\title{
Correction: Monitoring EGFR-T790M mutation in serum/plasma for prediction of response to third-generation EGFR inhibitors in patients with lung cancer
}

Teresa Morán ${ }^{1}$, Eudald Felip ${ }^{1}$, Joaquim Bosch-Barrera ${ }^{2}$, Itziar de Aguirre ${ }^{3}$, Jose Luis Ramirez ${ }^{3}$, Carles Mesia4, Enric Carcereny', Diana Roa ${ }^{2}$, Elia Sais², Yolanda García ${ }^{5}$, Remei Blanco6 ${ }^{6}$ Silvia Sanchez7, Claudia Rosa Villacorta7, Cristina Queralt ${ }^{3}$, Jose María Velarde ${ }^{8}$ and Rafael Rosell ${ }^{3}$

1 Medical Oncology Department, Catalan Institute of Oncology - Badalona, Hospital Universitari Germans Trias i Pujol, Badalona, Department of Medicine, Universitat Autònoma de Barcelona (UAB), Barcelona, Spain

2 Medical Oncology Department, Catalan Institute of Oncology-Girona, Hospital Universitari Doctor Trueta Girona, Girona, Spain

3 Molecular Biology Laboratory of Cancer Dr. Rosell, Can Ruti Campus: Institute Germans Trias i Pujol (IGTP), Catalan Institute of Oncology, Badalona, Spain

${ }^{4}$ Medical Oncology Department, Catalan Institute of Oncology - Hospital Duran i Reynalds, I'Hospitalet de Llobregat, Barcelona, Spain

${ }^{5}$ Medical Oncology Department, Hospital Parc Taulí, Sabadell, Barcelona, Spain

${ }^{6}$ Medical Oncology Department, Consorci Sanitari de Terrassa, Terrassa, Barcelona, Spain

${ }^{7}$ Research Nurse Team, Catalan Institute of Oncology - Badalona, Hospital Universitari Germans Trias i Pujol, Badalona, Spain

${ }^{8}$ Statistics Department, Fundació Germans Trias i Pujol, Badalona, Spain

${ }^{9}$ Department of Medicine, Universitat Autònoma de Barcelona (UAB), Barcelona, Spain

Published: April 02, 2019

Copyright: Morán et al. This is an open-access article distributed under the terms of the Creative Commons Attribution License 3.0 (CC BY 3.0), which permits unrestricted use, distribution, and reproduction in any medium, provided the original author and source are credited.

This article has been corrected: The correct affiliation information is given below:

1 Medical Oncology Department, Catalan Institute of Oncology - Badalona, Hospital Universitari Germans Trias i Pujol, Badalona, Department of Medicine, Universitat Autònoma de Barcelona (UAB), Barcelona, Spain

Original article: Oncotarget. 2018; 9:27074-27086. https://doi.org/10.18632/oncotarget.25478 